\title{
Effects of melting process on the hydromagnetic wedge flow of a Casson nanofluid in a porous medium
}

\author{
Subharthi Sarkar ${ }^{1}$ and Mehari Fentahun Endalew ${ }^{1,2^{*}}$
}

\section{"Correspondence:}

mehexf@gmail.com

'Department of Mathematics,

Kalinga Institute of Industrial

Technology, Bhubaneswar, India

${ }^{2}$ Department of Mathematics,

Debre Tabor University, Debre

Tabor, Ethiopia

\section{Springer}

\begin{abstract}
An investigation is carried out to capture the influence of melting process and permeability of the medium on the hydromagnetic wedge flow of a Casson nanofluid. The effects of thermophoresis and Brownian motion are also taken into consideration. The coupled nonlinear partial differential equations that govern the nanofluid flow are reduced to coupled non-linear ordinary differential equations by employing similarity transformation. Thereafter, numerical solution of the resulting boundary value problem is obtained using a fourth-order accurate collocation based solver in MATLAB. A particular case of the present problem is compared with a previously published study, and the results are found to be in excellent agreement. The impact of pertinent physical entities on nanofluid velocity, nanofluid temperature, and nanoparticle concentration are presented graphically, while local skin friction, heat transfer, and mass transfer rates are recorded in a tabular form. It is found that melting process increases the thicknesses of momentum, thermal and solutal boundary layers while it reduces skin friction, heat and mass transfer rates. Wedge angle and Casson nanofluid parameters enhance the fluid velocity; however, the impact of the magnetic field and permeability of the medium is opposite to that of their usual characteristics. This study would be valuable in designing cooling gadgets and heat sinks of various shapes which will enhance the heat transfer properties of Casson nanofluids thereby increasing their applications in industrial perspectives.
\end{abstract}

Keywords: Melting process; Casson nanofluid; Forced convection; Porous medium; Magnetohydrodynamic flows

\section{Introduction}

Boundary layer flow past a wedge is a classic problem in fluid dynamics, and its presence could be seen almost everywhere wherever general fluid flow takes place, be it industrial processes, manufacturing units, or the design of prototypes for technological advancements in aerospace or defense laboratories. For example, applications of flows past a wedge could be found in polymer processing, crude oil extraction, flow of molten metals over ramped surfaces, liquid metal flows in heat exchangers, throwing of chilled air through AC panels, nuclear power plants, designing flaps on airplane wings for enhanced lift, drag, and manoeuvre, modeling of warships, submarines, and in several other domains of sci-

(c) The Author(s) 2019. This article is distributed under the terms of the Creative Commons Attribution 4.0 International License (http://creativecommons.org/licenses/by/4.0/), which permits unrestricted use, distribution, and reproduction in any medium, provided you give appropriate credit to the original author(s) and the source, provide a link to the Creative Commons license, and indicate if changes were made. 
ence and engineering. In fact, wedge angle plays a crucial role in the study of transonic flows over airfoils and wings, including flows at Mach 1 as discussed by Jameson [1].

The classical boundary layer problems are expressed in terms of partial differential equations (PDEs). It is well known that PDEs have very important applications in engineering and sciences. Their significant impacts in real life problems are exhaustively discussed in the work of Ghergu and Radulescu [2] and Cavalcanti et al. [3]. The reduction of classical boundary layer equations into an ordinary differential equation via similarity transformation with exhaustive justification and explanation was made by Hartree [4]. Here, the equation contains external flow of the form $a x^{m}$ which is evaluated at the wall, where $x$ stands for coordinate axis along the wedge wall, and $a>0$ and $m$ are real constants. This equation came to be known as Falkner-Skan equation (named after V.M. Falkner and Sylvia W. Skan). Then, analytical solution with algebraic decay velocity field was found by Fang [5]. Later, Weyl [6] performed the mathematically rigorous analysis for the FalknerSkan equation. Recently, many scholars have demonstrated their distinct fascination in the investigation of fluid flow past a wedge surface. For instance Ashraf et al. [7], Ahmad [8], Hossain [9], and Ishak et al. [10] have investigated flows past a wedge with Dirichlet and Robin or mixed type thermal boundary conditions.

Fluids that contain small nanometer-sized particles (molecules or atoms) suspended in a base fluid are called nanofluids. Fluids like water, engine oil, and ethylene glycol with less thermal conductivity are known as base fluids. They are assumed as basic for heat transfer coefficient between the heat transfer of medium and surface. According to the experimental demonstrations, nanofluids have more thermal conductivity than base fluids. Historically, Choi and Eastman [11] invented nanofluids in 1995. They pointed out that presence of suspended nanoparticles enhanced the effective thermal conductivity of the base fluid. Convective transport in nanofluids was discussed in detail by Buongiorno [12]. We know that nanofluids with rheological characteristics in shear stress-strain relationship are called Casson nanofluids which belong to the part of non-Newtonian fluids. These fluids act as an elastic solid at low shear strain and behave as a Newtonian fluid above the critical stress value. The global existence of classical solution to a compressible nonNewtonian fluid was discussed by Fang et al. [13]. Some common Casson fluids are orange juice, soup, tomato sauce, honey, and blood. Furthermore, fluid flows under the influence of magnetic fields are of high significance in the study of geophysical, astrophysical phenomena and are routinely used in several processes in engineering and technology. Such flows are called magnetohydrodynamic (MHD) flows and have received phenomenal attention from world researchers owing to their infinitely many possible applications. History of works and applications in MHD flows can be found in Molokov et al. [14] and Sutton and Sherman [15]. Significant studies on the Cauchy problem for the generalized MHD system is carried out by Gala and Ragusa [16, 17]. In recent years, MHD flows with mass and heat transfer through porous media have been considered widely because of their crucial applications in various engineering processes such as filtration of liquid metals, casting, metallurgy, fusion control, cooling of nuclear reactors, and so on. However, till date there are too few investigations on MHD Casson nanofluid flows in a porous or permeable medium. Some of the related literatures are Buryachenko and Skrypnik [18], Kafoussias and Nanousis [19], Ullah et al. [20], and Mustafa and Khan [21].

It is well known that melting is driven from the physical change of a body by means of heating process. It results in the phase transition of a substance from a solid state to a liquid 
state. The melting problem has been a subject of highly demanding theoretical and experimental examinations for a long period of time. This is due to its close relationship with a vast variety of technologically imperative procedures. Some vital real-life applications are cooling and heating process, melting of permafrost, thermal energy storage, unfreezing of frozen grounds, setting up of semiconductor-material, casting and welding of manufacturing processes, and so on. Accordingly, many studies have been performed based on the melting. For instance, Roberts [22] studied the melting phenomenon of ice put in a hot stream of air at a steady state. The relationship between diffusion heat transfer and melting was initially investigated by Yen and Tien [23]. Epstein and Cho [24] discussed melting in steady laminar flow on the flat plate. An analysis of melting with natural convection at the melt region was performed by Sparrow et al. [25]. Recently, many researchers have studied boundary layer problems with melting heat transfer with different aspects, viz. Ishak et al. [26], Ibrahim [27], Abdel-Rahman [28], Ahmad and Pop [29], Yacob [30], and so on.

However, in all the above studies, no investigation is conducted on the effects of the combination of both wedge angle and melting process on MHD Casson nanofluid flow through a porous medium. Due to this reason, the authors were motivated to model and study the problem. The effects of different physical entities on nanofluid velocity, nanofluid temperature, nanoparticle concentration, local skin friction, heat transfer rate, and mass transfer rate are illustrated through the graphs and in a tabular form, respectively. All results are interpreted with physical rationale. Specifically, this investigation would be highly significant in designing cooling gadgets and heat sinks of various shapes which will enhance the heat transfer properties of Casson nanofluids thereby increasing their applications in industrial perspectives.

\section{Mathematical formulation}

Consider a forced convection steady two-dimensional incompressible Casson nanofluid flow past a wedge embedded through a porous medium with melting process. The induced magnetic field is assumed to be small and is thus neglected. Nanoparticle volumetric fraction and the temperature at the surface are denoted by $C_{w}$ and $T=T_{m}$, respectively. Here $T_{m}$ is the surface temperature due to melting. Further, the external (free stream) velocity of the nanofluid is considered as $U(x)=a x^{m}$, where $a>0$ denotes a real constant. Moreover, the magnetic field is assumed to be $B(x)=B_{o} x^{m}$, where $B_{o}$ is the constant magnetic field $[8,10]$ and applied normal to the wedge walls (as shown in Fig. 1). Similarly, the variable permeability of the medium is assumed as $K(x)=K^{\prime} x^{m}$, where $K^{\prime}$ is the di-

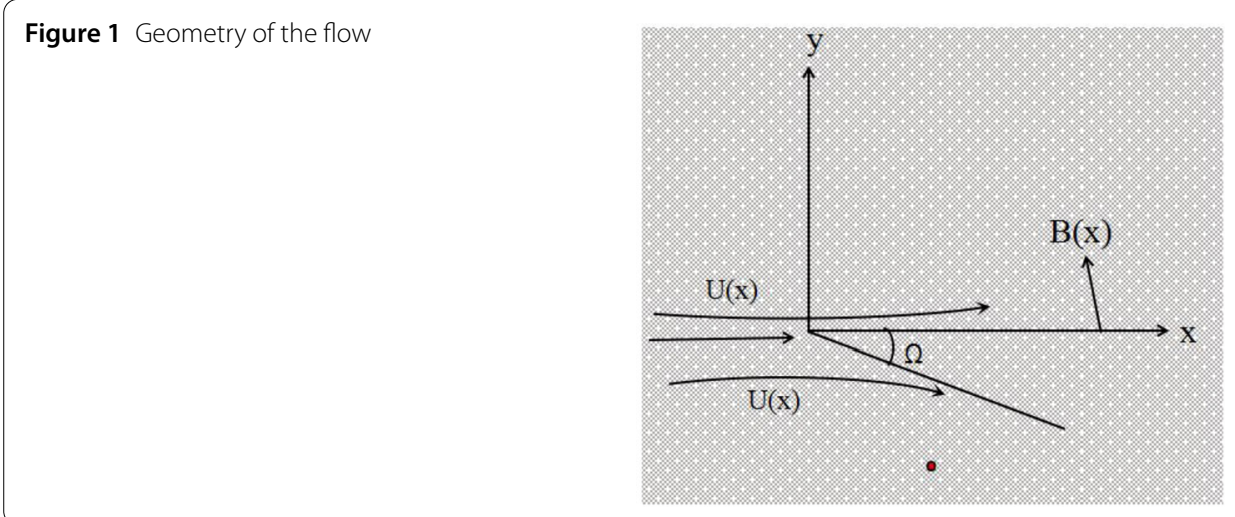


mensionless permeability and $m=\frac{\beta_{1}}{2-\beta_{1}}$ such that $\beta_{1}=\frac{\Omega}{\pi}$. Here $m, \beta_{1}$, and $\Omega$ stand for the wedge angle parameter, Hartree pressure gradient, and wedge angle, respectively [10]. We assumed that $m \in[0,1]$, in which $m=0$ stands for the horizontal plate and $m=1$ stands for the vertical plate. Temperature and the nanoparticle concentration far from the surface are given by $T_{\infty}$ and $C_{\infty}$, respectively. Here, $T_{m}<T_{\infty}$ and $T_{m}>T_{o}$. Having in mind all the above assumptions, nanofluid flow is governed by the following nonlinear partial differential equations:

$$
\begin{aligned}
& \frac{\partial u}{\partial x}+\frac{\partial v}{\partial y}=0, \\
& u \frac{\partial u}{\partial x}+v \frac{\partial u}{\partial y}=U(x) \frac{\partial U(x)}{\partial x}+v\left(1+\frac{1}{\beta}\right) \frac{\partial^{2} u}{\partial y^{2}}+\left(\frac{\sigma B^{2}(x)}{\rho_{f}}+\frac{v}{K(x)}\right)(U(x)-u), \\
& u \frac{\partial T}{\partial x}+v \frac{\partial T}{\partial y}=\alpha \frac{\partial^{2} T}{\partial y^{2}}+\tau\left\{D_{B} \frac{\partial C}{\partial y} \frac{\partial T}{\partial y}+\frac{D_{T}}{T_{\infty}}\left(\frac{\partial T}{\partial y}\right)^{2}\right\} \\
& u \frac{\partial C}{\partial x}+v \frac{\partial C}{\partial y}=D_{B} \frac{\partial^{2} C}{\partial y^{2}}+\frac{D_{T}}{T_{\infty}} \frac{\partial^{2} T}{\partial y^{2}} .
\end{aligned}
$$

In the above equations, $(u, v)$ are the velocity components, $T$ is the dimensional nanofluid temperature, $C$ is the dimensional nanoparticle concentration, $D_{B}$ denotes the Brownian diffusion, $D_{T}$ stands for the thermophoresis diffusion coefficient, $v$ is the nanofluid viscosity, $\alpha=\frac{k}{(\rho c)_{f}}$ denotes the thermal diffusivity, $\tau=\frac{(\rho c)_{p}}{(\rho c)_{f}}$ is the ratio of nanoparticle heat capacity to the base fluid, $\sigma$ is the electrical conductivity and $\beta$ is the Casson nanofluid parameter. Equation (1) is the continuity equation of incompressible nanofluid and Equation (2) describes the nanofluid momentum equation. Equation (3) states that the transport of heat in the nanofluid occurred by forced convection (first and second terms of LHS) and behavior of nanoparticle diffusion (second and third terms of RHS). Equation (4) states that the nanoparticles can not only move homogeneously within due to the convective terms (first and second term on the LHS), but are also affected by Brownian diffusion and thermophoresis (second and third term on RHS). Hence, the governing equations are highly coupled.

The boundary conditions for the nanofluid flow are given as follows [26, 29]:

$$
\begin{aligned}
& v=0, \quad u=0, \quad k \frac{\partial T}{\partial y}=\rho\left[\lambda+C_{s}\left(T_{m}-T_{o}\right)\right] v(x, 0), \\
& T=T_{m}, \quad C=C_{w} \quad \text { at } y=0, \\
& u \rightarrow U(x), \quad v=0, \quad T \rightarrow T_{\infty}, \quad C \rightarrow C_{\infty} \quad \text { as } y \rightarrow \infty .
\end{aligned}
$$

Here, $k, \lambda, T_{o}, \rho$, and $C_{s}$ are the thermal conductivity of the nanofluid, latent heat of the nanofluid, solid surface temperature, density and heat capacity of the solid surface, respectively. We introduce the similarity and dimensionless variables as follows [8, 26, 29]:

$$
\begin{aligned}
& \psi=\sqrt{\frac{2 x v U(x)}{m+1}} f(\eta), \quad \eta=\sqrt{\frac{(m+1) U(x)}{2 x v}}, \\
& \theta(\eta)=\frac{T-T_{m}}{T_{\infty}-T_{m}}, \quad \phi(\eta)=\frac{C-C_{\infty}}{C_{w}-C_{\infty}} .
\end{aligned}
$$


Let us define the stream function $\psi(x, y)$ as follows:

$$
v=-\frac{\partial \psi}{\partial x}, \quad u=\frac{\partial \psi}{\partial y} .
$$

For this choice of stream function, the continuity equation given in Equation (1) is satisfied. These stream functions given in Eq. (7) reduce Eqs. (2-4) as follows:

$$
\begin{aligned}
& \left(1+\frac{1}{\beta}\right) f^{\prime \prime \prime}+\frac{2 m}{m+1}\left(1-f^{\prime 2}\right)+f^{\prime \prime}+\left(M+\frac{1}{K_{p}}\right)\left(1-f^{\prime}\right)=0, \\
& \theta^{\prime \prime}+P_{r}\left\{N_{b} \phi^{\prime} \theta^{\prime}+f \theta^{\prime}+N_{t} \theta^{2}\right\}=0, \\
& \phi^{\prime \prime}+\frac{N_{t}}{N_{b}} \theta^{\prime \prime}+L e \phi^{\prime} f=0 .
\end{aligned}
$$

The corresponding boundary conditions are given:

$$
\begin{aligned}
& B \theta^{\prime}(\eta)+P_{r} f(\eta)=0, \quad f^{\prime}(\eta)=0, \quad \theta(\eta)=0, \quad \phi(\eta)=1 \quad \text { at } \eta=0, \\
& f^{\prime}(\eta) \rightarrow 1, \quad \theta(\eta) \rightarrow 1, \quad \phi(\eta) \rightarrow 0 \quad \text { as } \eta \rightarrow \infty .
\end{aligned}
$$

Here, the physical parameters that govern the flow are defined as follows:

$$
\begin{aligned}
& M=\frac{2 \sigma B_{o}^{2}}{\rho_{f} a(m+1)}, \quad K_{p}=\frac{a K^{\prime}(m+1)}{2 v}, \quad P_{r}=\frac{v}{\alpha}, \quad B=\frac{C_{f}\left(T_{m}-T_{o}\right)}{\lambda+C_{s}\left(T_{m}-T_{o}\right)}, \\
& N_{t}=\frac{D_{T}\left(T_{m}-T_{\infty}\right) \tau}{v T_{\infty}}, \quad N_{b}=\frac{D_{B}\left(C_{w}-C_{\infty}\right) \tau}{v}, \quad L e=\frac{v}{D_{B}},
\end{aligned}
$$

where $f^{\prime}, \theta, \phi, P r, L e, N b, N t, M$, and $K_{p}$ are the dimensionless nanofluid velocity, nanofluid temperature, nanoparticle concentration, Prandtl number, Lewis number, Brownian parameter, thermophoresis parameter, magnetic field parameter, and permeability parameter, respectively. The melting parameter $B$ is defined as $B=\frac{C_{p} f\left(T_{m}-T_{o}\right)}{\lambda+C_{S}\left(T_{m}-T_{o}\right)}$, here, $\frac{C_{p} f\left(T_{m}-T_{o}\right)}{\lambda}$ and $\frac{C_{s}\left(T_{m}-T_{o}\right)}{\lambda}$ are the Stefan numbers for the liquid state and solid state, respectively $[8$, 29].

Next, depending on the above quantities, the skin friction, Nusselt and Sherwood numbers are given in a dimensionless form as follows:

$$
\sqrt{R e_{x}} C f_{x}=\left(1+\frac{1}{\beta}\right) f^{\prime \prime}(0), \quad \frac{N u}{\sqrt{R e_{x}}}=-\theta^{\prime}(0), \quad \frac{S h}{\sqrt{R e_{x}}}=-\phi^{\prime}(0),
$$

where $\operatorname{Re}_{x}, C_{f} x, N u, C_{p} f$, and $S h$ are the local Reynolds number, skin friction, Nusselt number, specific heat capacity of convective fluid, and Sherwood number, respectively.

\section{Numerical solution}

The partial differential Eqs. (1)-(4) that govern the nanofluid flow problem are transformed to nonlinear coupled ordinary differential equations (ODEs) by employing boundary layer theory. These highly nonlinear coupled ordinary differential Eqs. (8)-(10) with respect to the boundary conditions (11a), (11b) are solved numerically by calling the famous BVP4C solver of MATLAB. The BVP4C solver is based on a 3-stage Lobatto IIIa collocation formula whose collocation polynomial provides a $\mathrm{C} 1$-continuous solution which 
Table 1 Comparison of skin friction $f^{\prime \prime}(0)$ when $P_{r}=1$ for different $B$ with existing result

\begin{tabular}{lll}
\hline$B$ & Ishak et al. [26] & Present result \\
\hline O(Blasius) & 0.4696 & 0.4696 \\
1 & 0.2786 & 0.2790 \\
2 & 0.2019 & 0.2022 \\
3 & 0.1594 & 0.1598 \\
\hline
\end{tabular}

is fourth-order accurate throughout the entire domain [31]. In order to validate the correctness of our numerical computation, we have run the same numerical code in the absence of wedge angle, Casson nanofluid parameter, permeability, and magnetic field. Then we have extracted the values of skin friction corresponding to $\epsilon=0$ from Fig. 1 of Ishak et al. [26]. After that we have compared our values of skin friction with those of Ishak et al. [26] and have presented them in Table 1. We found that the results are in excellent agreement with maximum percentage error to be only $0.001 \%$. This justifies the correctness of our numerical results.

\section{Results and discussion}

In this problem, we propose to study the effects of melting and wedge angle parameters on the two-dimensional incompressible steady MHD Casson nanofluid flow past a wedge embedded in a porous medium. In this regard, the impacts of important physical entities on nanofluid velocity, nanofluid temperature, nanoparticle concentration, skin friction, heat transfer rate, and mass transfer rate are presented through Figs. 2-16 and Table 2, respectively. The values of physical parameters used for the numerical simulations of the graphs are, unless otherwise stated: $L e=1, P_{r}=1, \beta=10, M=1, N b=N t=m=K_{p}=0.5$, $B=1$.

Figure 2 shows the influence of the magnetic field parameter on nanofluid velocity. Here, we can see that the thickness of boundary layer diminishes with an increment in the magnetic field, i.e., nanofluid velocity tends to free stream velocity earlier for a greater strength of the field. This means that magnetic field enhances the magnitude of nanofluid velocity in this case. This is contrary to the usual nature of magnetic field to suppress the flow. This can be attributed to a positive body force term containing magnetic field on the right of the momentum Eq. (2). The positive sign of a body force originates from the fact that $U(x)>u$ in Eq. (2), i.e., free stream (external) velocity $(U(x))$ is more than the nanofluid velocity $(u)$ in the boundary layer. The impact of the permeability parameter on the nanofluid velocity is exhibited in Fig. 3. It is noted that nanofluid velocity reduces with an increment in permeability of the medium. This is why the nanofluid velocity reaches free stream velocity later for a higher value of $K_{p}$. The reverse trend of permeability on nanofluid velocity is akin to the previous effect of magnetic field on it.

The impact of wedge angle on the nanofluid velocity is shown in Fig. 4. It is noted from Fig. 4 that nanofluid velocity rises as the wedge angle parameter goes up. An increment in the wedge angle parameter enhances forced convection, which in turn adds the nanofluid velocity. The influence of the Casson nanofluid parameter on the nanofluid velocity is shown in Fig. 5. From this figure, we can see that as the Casson nanofluid parameter rises, the nanofluid velocity rises, while the thickness of boundary layer reduces. It can be easily perceived from Equation (2) that, with an increment in the value of Casson nanofluid parameter, the momentum equation tends to the momentum equation of a Newtonian fluid. Hence nanofluid velocity increases as the effective viscous drag force decreases with 
Figure 2 Nanofluid velocity for different values of $M$

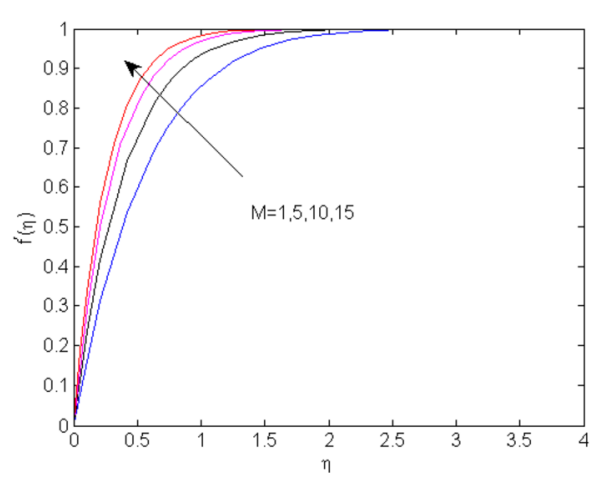

Figure 3 Nanofluid velocity for different values of $K_{p}$

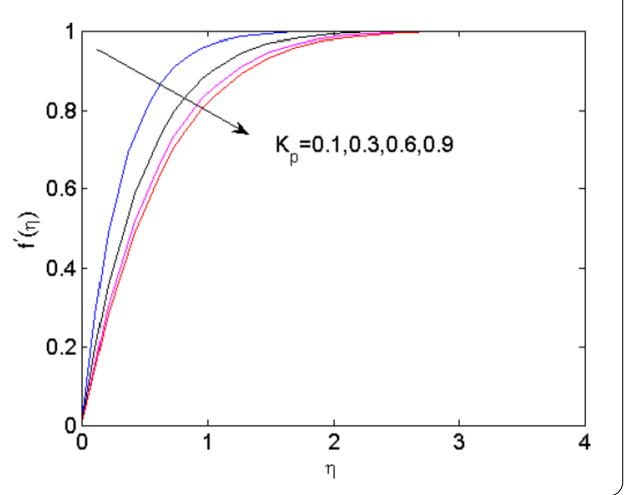

Figure 4 Nanofluid velocity for different values of $m$

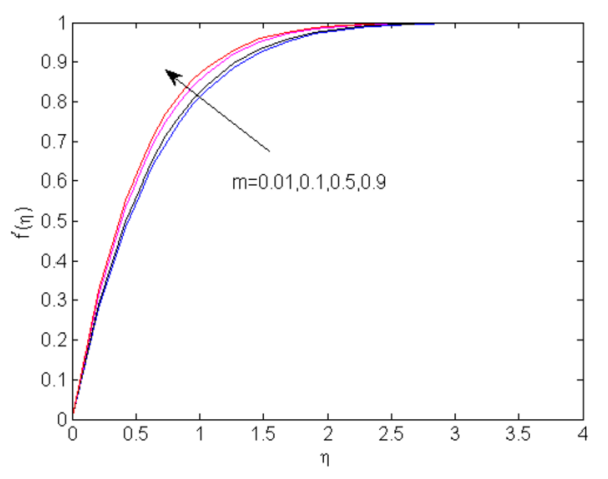

the increase in $\beta$, i.e., $v(1+1 / \beta) \ll v(1+1 / \beta)$ for higher values of $\beta$. This is why nanofluid velocity reaches the free stream velocity earlier for a greater value of $\beta$. The increase in $\beta$ also causes an increase in the surface shear stress or skin friction (Table 2), which in turn increases the thickness of boundary layer. This is in compliance with the previous findings of Mukhopadhyay et al. [32].

The impact of melting $(B)$ on nanofluid velocity is depicted in Fig. 6. It is observed that melting enhances the thickness of boundary layer, that is, velocity boundary layer, thermal boundary layer, and solutal boundary thicknesses increase with the increase in the melting parameter (as seen in Figs. 6, 7, and 12). Also, the melting parameter reduces the skin friction, Nusselt and Sherwood numbers, i.e., heat and mass transfer rates, are hindered by the melting process. This reduces forced convection current, and hence the nanofluid 
Figure 5 Nanofluid velocity for different values of $\beta$

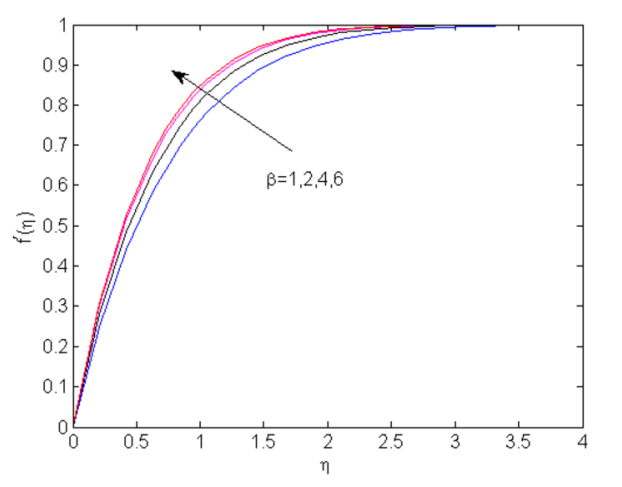

Figure 6 Nanofluid velocity for different values of $B$

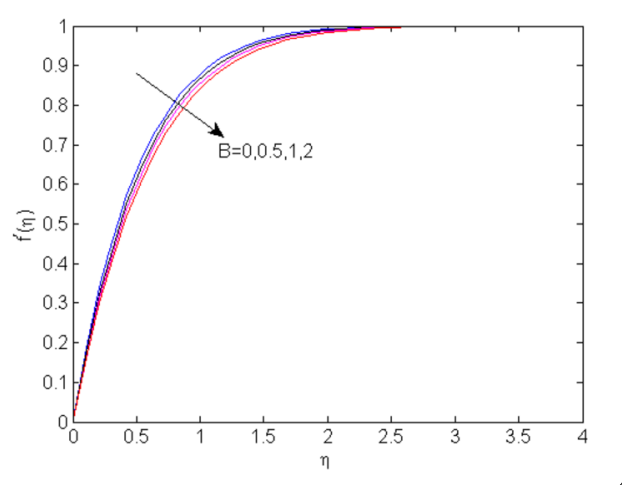

Figure 7 Nanofluid temperature for different values of $B$

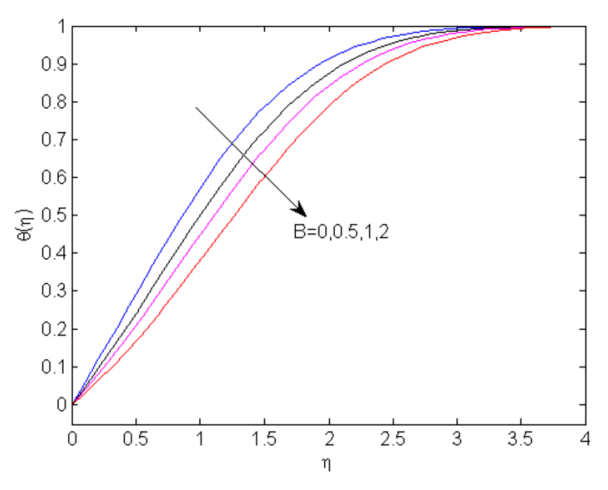

velocity is also reduced by this process. Physically, the outcome confirms that the liquefying under goes about like a blowing boundary condition at the extending wall. Figure 7 exhibits the impact of melting $B$ on the nanofluid temperature graph. When the melting parameter increases, the graph of nanofluid temperature decreases. Physically speaking, rising the parameter $B$ results in more acceleration to the nanofluid flow which, in turn, convects more heat energy into the ambiance and causes a reduction in the nanofluid temperature.

When the magnetic field increases, the nanofluid temperature increases as portrayed in Fig. 8. We have seen (see Fig. 2) that nanofluid velocity rises with an increment in the magnetic field. Hence, nanofluid temperature increases as a result of forced convection. Thickness of the thermal boundary layer reduces with the increment in the magnetic field. 


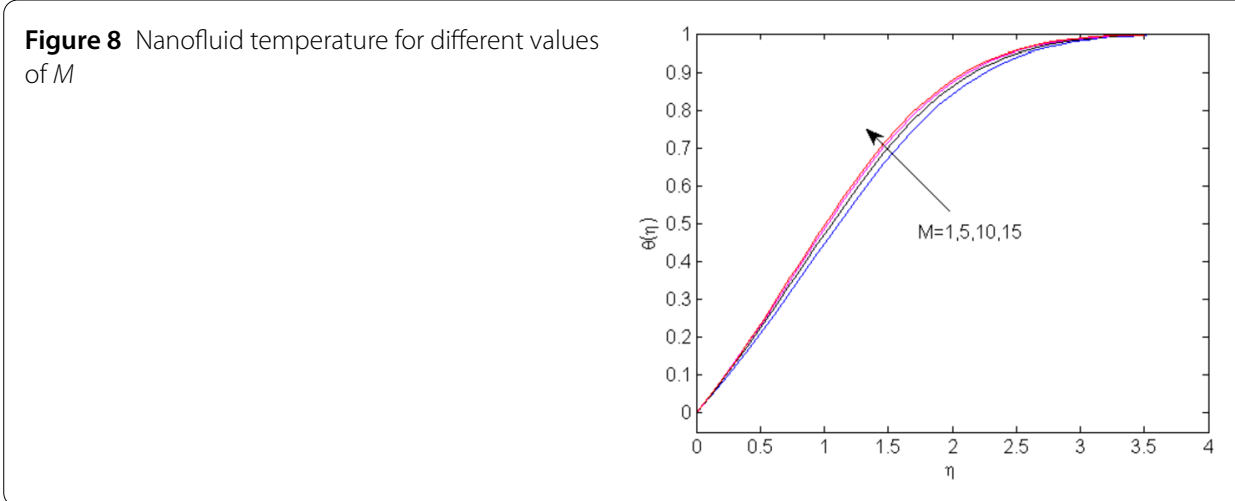

Figure 9 Nanofluid temperature for different values of $P_{r}$

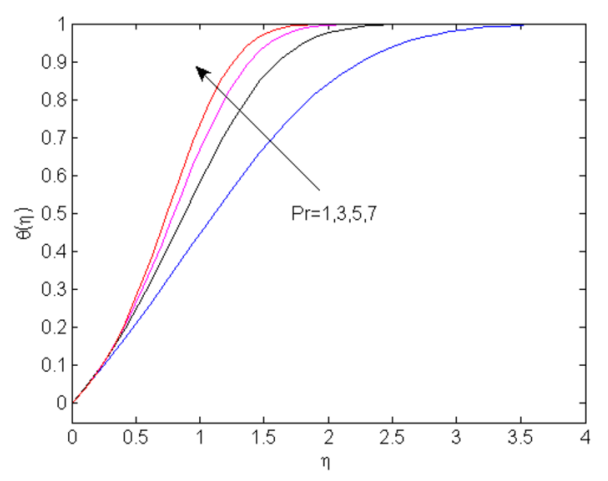

Figure 10 Nanofluid temperature for different values of $\mathrm{Nb}$

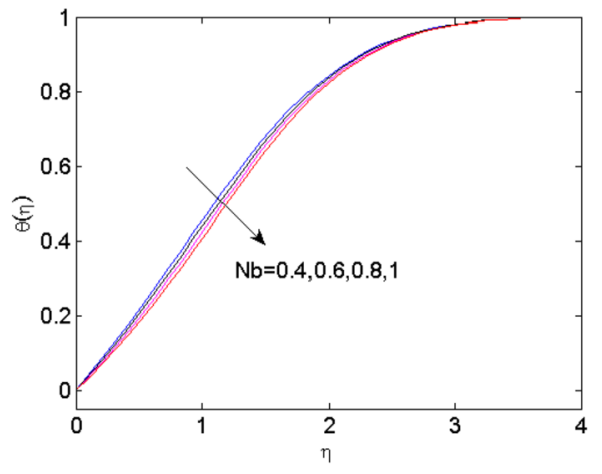

Figure 9 depicts the impact of Prandtl number on the nanofluid temperature. In the analysis of heat transfer, the relative thermal boundary layer thickness may be controlled by Prandtl number. Hence, when Prandtl number is large, heat diffuses slowly. This means that for a larger value of Prandtl number the thermal boundary layer thickness is much less. Hence, as Prandtl number increases thermal diffusion decreases, thus, inhibiting the melting process. This results in the increase in nanofluid temperature with Prandtl number. The influence of Brownian motion on the nanofluid temperature is shown in Fig. 10. From this figure, we can observe that, as $N_{b}$ increases, nanofluid temperature decreases. Physically, Brownian motion is unspecified movement of small particles (molecules or atoms) suspended in a fluid resulting from their collision with rapid motion of molecules 
Figure 11 Nanofluid temperature for different values of $\mathrm{Nt}$

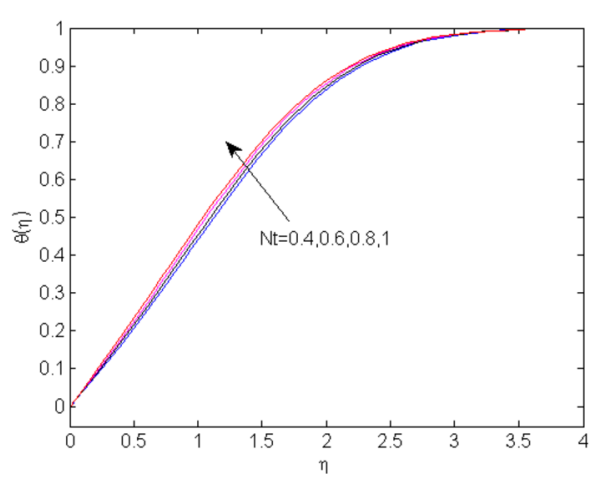

Figure 12 Nanoparticle concentration for different values of $B$

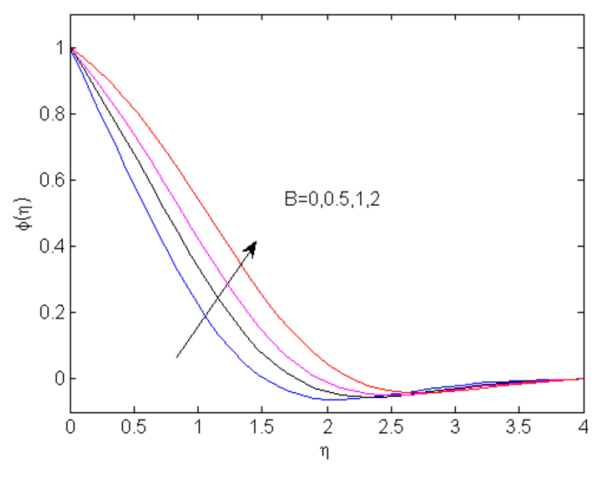

Figure 13 Nanoparticle concentration for different values of Le

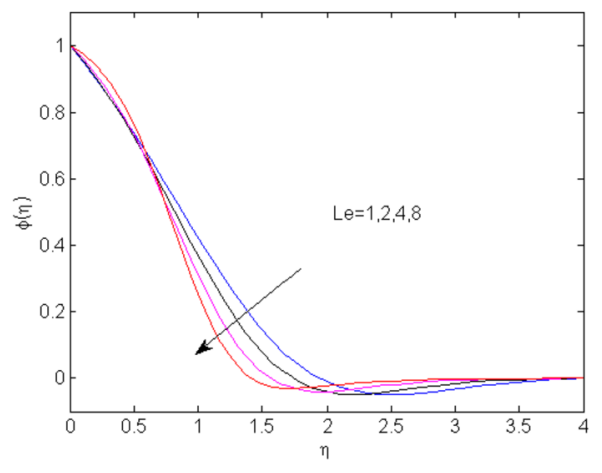

in the nanofluid. Such collision enhances the melting process which yields more nanofluid into the system resulting in the reduction in nanofluid temperature.

The impact of thermophoresis on the nanofluid temperature is visualized in Fig. 11. Here, we can see that the fluid temperature increases as the thermophoresis parameter increases. It is known that thermophoresis is the movement of molecules in the direction of positive temperature gradient. So, the suspended nanoparticles will tend to move from the regions of high temperature, i.e., from the free stream, to low temperature, i.e., the body of the melting surface. This reverse concentration gradient (from the free steam towards the surface) hinders the melting process by forming a barrier to the heat conduction process, thus increasing the overall temperature of the system. 
Figure 14 Nanoparticle concentration for different values of $M$

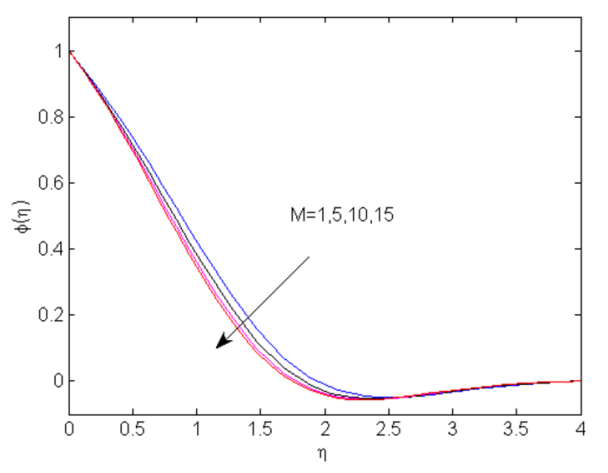

Figure 15 Nanoparticle concentration for different values of $\mathrm{Nb}$

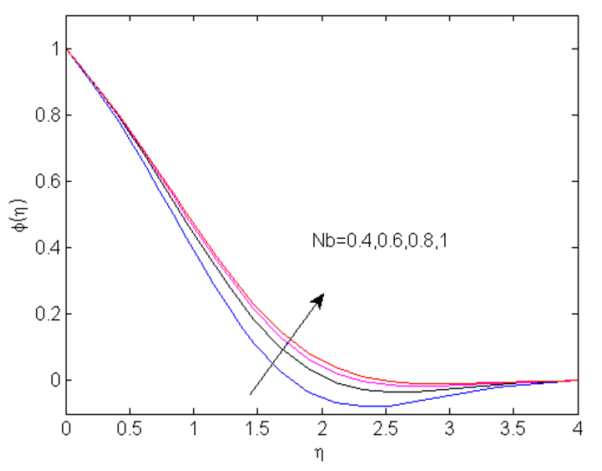

Figure 16 Nanoparticle concentration for different values of $\mathrm{Nt}$

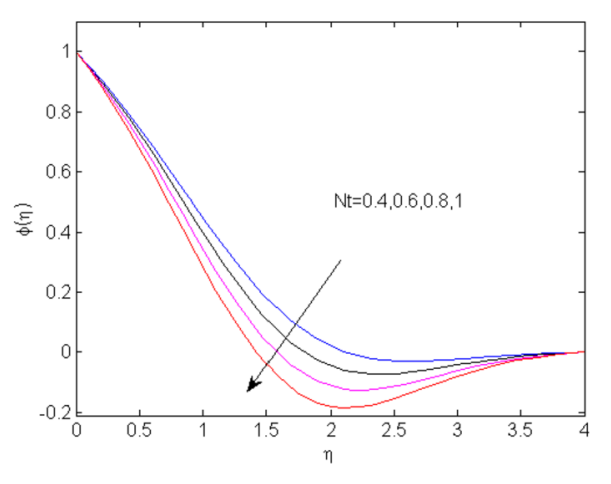

Figure 12 shows the impact of the melting parameter on the nanoparticle concentration. Here, we can see that nanoparticle concentration expands as the melting parameter rises (similar argument as presented for Fig. 6). Figure 13 depicts the influence of Lewis number on the nanoparticle concentration. When Lewis number goes up, the nanoparticle concentration diminishes in magnitude throughout the boundary layer region. Physically speaking, the quotient of thermal diffusivity to the mass diffusivity is known as Lewis number. Therefore, when Lewis number rises, the concentration of nanoparticles diminishes. This is due to the inverse proportionality of Lewis number and mass diffusivity. A reverse flow in nanoparticle concentration is observed at $\eta=2$ which may be attributed to the melting process. The impact of the magnetic parameter on the nanoparticle concentration is exhibited in Fig. 14. Here, we can see that the nanoparticle concentration 
Table 2 Variation of skin friction, Nusselt and Sherwood numbers for different parameters. In this table, the boldface values specify that we are increasing the values of those particular physical parameters keeping the values of other parameters fixed

\begin{tabular}{llllllllllll}
\hline$m$ & $\beta$ & $P_{r}$ & $N b$ & $N t$ & $M$ & $L e$ & $K p$ & $B$ & $f^{\prime \prime}(0)$ & $\theta^{\prime}(0)$ & $-\phi^{\prime}(0)$ \\
\hline $\mathbf{0 . 5}$ & 10 & 1 & 0.5 & 0.5 & 1 & 1 & 0.5 & 1 & 1.74873 & 0.37903 & 0.43844 \\
$\mathbf{0 . 6}$ & 10 & 1 & 0.5 & 0.5 & 1 & 1 & 0.5 & 1 & 1.77412 & 0.38005 & 0.44001 \\
0.5 & $\mathbf{1 1}$ & 1 & 0.5 & 0.5 & 1 & 1 & 0.5 & 1 & 1.75520 & 0.37928 & 0.43883 \\
0.5 & $\mathbf{1 2}$ & 1 & 0.5 & 0.5 & 1 & 1 & 0.5 & 1 & 1.76065 & 0.37949 & 0.43915 \\
0.5 & 10 & $\mathbf{2}$ & 0.5 & 0.5 & 1 & 1 & 0.5 & 1 & 1.74695 & 0.38312 & 0.40382 \\
0.5 & 10 & $\mathbf{3}$ & 0.5 & 0.5 & 1 & 1 & 0.5 & 1 & 1.75246 & 0.37052 & 0.37780 \\
0.5 & 10 & 1 & $\mathbf{0 . 6}$ & 0.5 & 1 & 1 & 0.5 & 1 & 1.75471 & 0.36538 & 0.43145 \\
0.5 & 10 & 1 & $\mathbf{0 . 7}$ & 0.5 & 1 & 1 & 0.5 & 1 & 1.76066 & 0.35183 & 0.42844 \\
0.5 & 10 & 1 & 0.5 & $\mathbf{0 . 6}$ & 1 & 1 & 0.5 & 1 & 1.74399 & 0.38990 & 0.44897 \\
0.5 & 10 & 1 & 0.5 & $\mathbf{0 . 7}$ & 1 & 1 & 0.5 & 1 & 1.73915 & 0.40103 & 0.46249 \\
0.5 & 10 & 1 & 0.5 & 0.5 & $\mathbf{2}$ & 1 & 0.5 & 1 & 1.96975 & 0.38605 & 0.44948 \\
0.5 & 10 & 1 & 0.5 & 0.5 & $\mathbf{3}$ & 1 & 0.5 & 1 & 2.17000 & 0.39165 & 0.45838 \\
0.5 & 10 & 1 & 0.5 & 0.5 & 1 & $\mathbf{2}$ & 0.5 & 1 & 1.75051 & 0.37498 & 0.41723 \\
0.5 & 10 & 1 & 0.5 & 0.5 & 1 & $\mathbf{3}$ & 0.5 & 1 & 1.75133 & 0.37311 & 0.38124 \\
0.5 & 10 & 1 & 0.5 & 0.5 & 1 & 1 & $\mathbf{0 . 6}$ & 1 & 1.66917 & 0.37626 & 0.43411 \\
0.5 & 10 & 1 & 0.5 & 0.5 & 1 & 1 & $\mathbf{0 . 7}$ & 1 & 1.61015 & 0.37410 & 0.43076 \\
0.5 & 10 & 1 & 0.5 & 0.5 & 1 & 1 & 0.5 & $\mathbf{2}$ & 1.66148 & 0.29195 & 0.27905 \\
0.5 & 10 & 1 & 0.5 & 0.5 & 1 & 1 & 0.5 & $\mathbf{3}$ & 1.60535 & 0.24056 & 0.19200 \\
\hline
\end{tabular}

diminishes with an increment in the magnetic field parameter. What is more, magnetic field in the fluid causes the reduction on the nanoparticle concentration. Effect of Brownian motion on the nanoparticle concentration is shown in Fig. 15. Here, we can see that, as the Brownian parameter increases, nanoparticle concentration increases throughout the flow domain. The impact of thermophoresis on the nanoparticle concentration is depicted in Fig. 16. Thus we conclude that, when thermophoresis increases, the nanoparticle concentration decreases.

\subsection{Discussion of local skin friction, Nusselt and Sherwood numbers}

The effects of all pertinent physical parameters on the local skin friction, Nusselt and Sherwood numbers are visualized in Table 2. From this table, we can see that the skin friction $\left(f^{\prime \prime}(0)\right)$ rises as $m, \beta, P_{r}, N b, M, L e$, and $K_{p}$ increase, whereas it reduces with the increment in $N t$ and $B$. This implies that skin friction expands with the increment in the wedge angle parameter, magnetic field, permeability of the medium, Brownian motion, Casson nanofluid parameter, and Lewis number, while it reduces with the increase in thermophoresis and melting parameters. Nusselt number $\left(\theta^{\prime}(0)\right)$ increases as $m, \beta, N t$, and $M$ increase, whereas it decreases with the increase in $N b, P_{r}, L e, K_{p}$, and $B$. That is, heat transfer rate increases as the wedge angle, Casson nanofluid parameter, thermophoresis, and magnetic field increase, whereas it reduces with the increment in the Brownian parameter, Prandtl number, Lewis number, permeability, and melting parameter. Sherwood number $\left(-\phi^{\prime}(0)\right)$ increases as $m, \beta, N t$, and $M$ increase, whereas it reduces with the increment in $N b, P_{r}, L e, K_{p}$, and $B$. This implies that mass transfer rate increases as the wedge angle, Casson nanofluid parameter, thermophoresis, and melting parameter increase. However, it reduces with increase in Brownian motion, Prandtl number, Lewis number, permeability, and melting parameter.

\section{Conclusion}

A numerical investigation has been carried out to examine the influences of melting and wedge angle parameters on the two-dimensional steady MHD Casson nanofluid flow with 
heat and mass transfer past a wedge embedded in a porous medium. Employing boundary layer theory, equations that govern the nanofluid flow were reduced to a system of highly nonlinear ordinary differential equations. Thereafter, the fourth-order accurate collocation based solver BVP4C is called in MATLAB to obtain the numerical solution of the resulting boundary value problem. The impacts of pertinent physical entities on the nanofluid velocity, temperature, nanoparticle concentration, local skin friction, heat transfer rate, and mass transfer rate are presented through graphs and a table. The following major conclusions are drawn from the investigation.

- The nanofluid velocity and local skin friction increase with increasing of magnetic field and decrease with increasing of the permeability parameter. This is due to the presence of positive body force.

- The nanofluid velocity increases with the increase in the wedge angle, Casson nanofluid, and melting parameters.

- The nanofluid temperature diminishes with the increment in the melting parameter, whereas it rises with the increase in magnetic field and Prandtl number.

- An increment in Lewis number causes the reduction in nanoparticle concentration in the interval $0.5 \leq \eta \leq 2$.

- Thickness of thermal boundary layer decreases as Prandtl number increases.

- The nanofluid temperature decreases as Brownian motion rises, whereas it increases as the thermophoresis parameter goes up.

- The nanoparticle concentration rises as Brownian motion increases, whereas it decreases as the thermophoresis parameter rises.

- The local skin friction and Nusselt number rise with increase in the Brownian and thermophoresis parameters, respectively.

- Nanoparticle (mass) transfer rate increases with increasing values of the thermophoresis parameter, wedge angle parameter, and Casson fluid, whereas it reduces with the increment in Brownian motion, permeability, and Lewis number.

\section{Acknowledgements}

Authors are thankful to the learned reviewers for their time and efforts in reviewing our article. The quality of the article has been highly enhanced via the help of the valuable and constructive comments/suggestions of them. Also, authors would like to thank Dr. A Nayak and Dr. M Das of the Department of Applied Mathematics, SAS, KIIT Bhubaneswar for the technical discussions and support.

Funding

The authors declare that no funding has been received to carry out this research work.

\section{Abbreviations}

MHD, Magnetohydrodynamic; PDE, Partial differential equation; ODE, Ordinary differential equation; LHS, Left-hand side of a given equation; RHS, Right-hand side of a given equation.

\section{Availability of data and materials}

We declare that the materials described in the manuscript, including all relevant raw data, will be freely available to any scientist wishing to use them for non-commercial purposes, without breaching participant confidentiality.

Competing interests

The authors declare that they have no competing interests.

Authors' contributions

The main idea of this paper was proposed by Dr. SS. MFE prepared the manuscript initially and performed all the steps of the solutions in this research. Both authors read and approved the final manuscript.

\section{Publisher's Note}

Springer Nature remains neutral with regard to jurisdictional claims in published maps and institutional affiliations. 


\section{References}

1. Jameson, A.: Iterative solution of transonic flows over airfoils and wings, including flows at Mach 1. Commun. Pure Appl. Math. 27(3), 283-309 (1974)

2. Ghergu, M., Radulescu, V.: Nonlinear PDEs: Mathematical Models in Biology, Chemistry and Population Genetics. Springer Monographs in Mathematics. Springer, Heidelberg (2012)

3. Cavalcanti, M.M., Cavalcanti, V.N.D., Lasiecka, I., Webler, C.M.: Intrinsic decay rates for the energy of a nonlinear viscoelastic equation modeling the vibrations of thin rods with variable density. Adv. Nonlinear Anal. 6(2), 121-145 (2017)

4. Hartree, D.R.: On an equation occurring in Falkner and Skan's approximate treatment of the equations of the boundary layer. In: Mathematical Proceedings of the Cambridge Philosophical Society, vol. 33, pp. 223-239. Cambridge University Press, Cambridge (1937)

5. Fang, T., Yao, S., Zhang, J., Zhong, Y., Tao, H.: Momentum and heat transfer of the Falkner-Skan flow with algebraic decay: an analytical solution. Commun. Nonlinear Sci. Numer. Simul. 17(6), 2476-2488 (2012)

6. Weyl, H.: On the differential equations of the simplest boundary-layer problems. Ann. Math. 43, 381-407 (1942)

7. Ashraf, M., Narahari, M., Muthuvalu, M.S.: Mixed convection flow over a stretching porous wedge with Newtonian heating in the presence of heat generation or absorption. In: AIP Conference Proceedings, vol. 1787, p. 020004. AIP, New York (2016)

8. Ahmad, K., Hanouf, Z., Ishak, A.: MHD Casson nanofluid flow past a wedge with Newtonian heating. Eur. Phys. J. Plus 132(2), $87(2017)$

9. Hossain, M.A., Roy, N.C., Siddiqa, S.: Unsteady mixed convection dusty fluid flow past a vertical wedge due to small fluctuation in free stream and surface temperature. Appl. Math. Comput. 293, 480-492 (2017)

10. Ishak, A., Nazar, R., Pop, I.: MHD boundary-layer flow of a micropolar fluid past a wedge with variable wal temperature. Acta Mech. 196(1-2), 75-86 (2008)

11. Chol, S., Estman, J.: Enhancing thermal conductivity of fluids with nanoparticles. ASME-Publications-Fed. 231, 99-106 (1995)

12. Buongiorno, J.: Convective transport in nanofluids. J. Heat Transf. 128(3), 240-250 (2006)

13. Fang, L., Zhu, H., Guo, Z.: Global classical solution to a one-dimensional compressible non-Newtonian fluid with large initial data and vacuum. Nonlinear Anal. 174, 189-208 (2018)

14. Molokov, S.S., Moreau, R., Moffatt, H.K.: Magnetohydrodynamics: Historical Evolution and Trends, vol. 80. Springer, Berlin (2007)

15. Sutton, G.W., Sherman, A.: Engineering Magnetohydrodynamics. Dover, New York (2006)

16. Gala, S., Ragusa, M.A.: Note on the blow-up criterion for generalized MHD equations. In: AIP Conference Proceedings, vol. 1798, p. 020058. AIP, New York (2017)

17. Gala, S., Ragusa, M.A.: A note on regularity criteria in terms of pressure for the 3D viscous MHD equations. Math. Notes 102(3-4), 475-479 (2017)

18. Buryachenko, K.O., Skrypnik, I.I.: Riesz potentials and pointwise estimates of solutions to anisotropic porous medium equation. Nonlinear Anal. 178, 56-85 (2019)

19. Kafoussias, N., Nanousis, N.: Magnetohydrodynamic laminar boundary-layer flow over a wedge with suction or injection. Can. J. Phys. 75(10), 733-745 (1997)

20. Ullah, I., Khan, I., Shafie, S.: MHD natural convection flow of Casson nanofluid over nonlinearly stretching sheet through porous medium with chemical reaction and thermal radiation. Nanoscale Res. Lett. 11(1), 527 (2016)

21. Mustafa, M., Khan, J.A.: Model for flow of Casson nanofluid past a non-linearly stretching sheet considering magnetic field effects. AIP Adv. 5(7), 077148 (2015)

22. Roberts, L.: On the melting of a semi-infinite body of ice placed in a hot stream of air. J. Fluid Mech. 4(5), 505-528 (1958)

23. Yen, Y.C., Tien, C.: Laminar heat transfer over a melting plate, the modified Leveque problem. J. Geophys. Res. 68(12), 3673-3678 (1963)

24. Epstein, M., Cho, D.: Melting heat transfer in steady laminar flow over a flat plate. J. Heat Transf. 98(3), $531-533$ (1976)

25. Sparrow, E., Patankar, S., Ramadhyani, S.: Analysis of melting in the presence of natural convection in the melt region. J. Heat Transf. 99(4), 520-526 (1977)

26. Ishak, A., Nazar, R., Bachok, N., Pop, I.: Melting heat transfer in steady laminar flow over a moving surface. Heat Mass Transf. 46(4), 463-468 (2010)

27. Ibrahim, W.: Magnetohydrodynamic (MHD) boundary layer stagnation point flow and heat transfer of a nanofluid past a stretching sheet with melting. Propuls. Power Res. 6(3), 214-222 (2017)

28. Abdel-Rahman, R.G., Khader, M., Megahed, A.M.: Melting phenomenon in magneto hydro-dynamics steady flow and heat transfer over a moving surface in the presence of thermal radiation. Chin. Phys. B 22(3), 030202 (2013)

29. Ahmad, S., Pop, I.: Melting effect on mixed convection boundary layer flow about a vertical surface embedded in a porous medium: opposing flows case. Transp. Porous Media 102(3), 317-323 (2014)

30. Yacob, N.A., Ishak, A., Pop, I.: Melting heat transfer in boundary layer stagnation-point flow towards a stretching/shrinking sheet in a micropolar fluid. Comput. Fluids 47(1), 16-21 (2011)

31. Kierzenka, J., Shampine, L.F.: A BVP solver based on residual control and the Matlab PSE. ACM Trans. Math. Softw. 27(3), 299-316 (2001)

32. Mukhopadhyay, S., Mondal, I.C., Chamkha, A.J.: Casson fluid flow and heat transfer past a symmetric wedge. Heat Transf. Asian Res. 42(8), 665-675 (2013) 